\title{
Alzheimer disease PS-1 exon 9 deletion defined
}

To the editor-The clinical, pathological, and genetic analyses of a Finnish pedigree (Finn2) with early-onset inherited Alzheimer disease (AD) have been reported $^{1}$. This family demonstrated linkage to the PS1 region of chromosome 14, and analysis for PS1 mutations by RT-PCR and immunoblot demonstrated heterozygous deletion of exon 9, which has been shown to cause early-onset $\mathrm{AD}^{2}$. However, unlike a previously described family ${ }^{2}$, no splice acceptor site mutation was detected in Finn2, suggesting the presence of either a small genomic deletion that included exon 9 or of mutations elsewhere that could affect splicing.

Intronic primers flanking exon 9 were designed using the PAC sequence available in the Genbank sequence database (clone DJ0054D12; accession number AC006342). PCR amplification of an approximate $6.5-\mathrm{kb}$ region encompassing exon 9 showed the presence of an aberrant product that segregated with affected members of Finn2 (Fig. 1) and was not present in more than 150 alleles of a

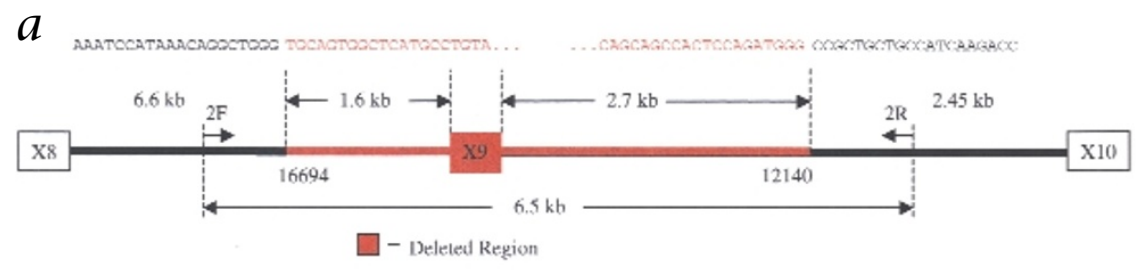

$b$

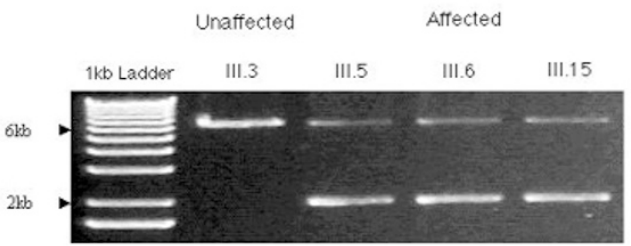

Fig. 1 Genomic analysis of PS-1. PCR used intronic primers (2F, 5'-TTTAAATCTGCATATITCCAGCCAGGCATGAC-3'; 2R, 5'-AAAGCATTAGGTCTCATCCTTTAGTGCACG-3') flanking exon 9, with the Expand Long PCR System. Cycling conditions were as described by the manufacturer (Hybaid Touchdown thermocycler, cycling conditions were as described by the manufacturer, except that a 'touchdown' program was used such that annealing temperatures gradually decreased from $62{ }^{\circ} \mathrm{C}$ to $55.1^{\circ} \mathrm{C}$ over 30 cycles. The initial extension time was 4 min $15 \mathrm{~s}$ and was increased by 20 s/cycle beginning on the $11^{\text {th }}$ cycle. Short PCR products (from affected individuals) and long PCR products derived from both affected and non-affected individuals in Finn2 were excised from lowmelting-point agarose gel (FMC), purified using the QIAquick PCR Purification System (Qiagen), and sequenced on $A B I 377$ (Perkin Elmer) using Big Dye chemistry. Primers used for sequencing (OAPS1 R4, 5'-CTITCTATCAGGTTTACTAGTGGTCTTGATGGCAGC-3'; OAPS1F4, 5'-GATGTAAGGTGTTTGTGTTAAGGTGAAATGAAAAGTGA-3') flanked deleted sequence. $\boldsymbol{a}$, Sequence proximal to exon 9 and the relative positions of primers $2 \mathrm{~F}$ and $2 \mathrm{R}$ are shown. Nucleotide numbers at the deletion points are based on PAC sequence (accession number AC006342; clone number DJ0054D12). X8 and X10 are PS-1 exons 8 and 10 respectively. $\boldsymbol{b}$, PCR results showing the heterozygous deletion of the 4,555-bp region encompassing exon 9 in affected family members of Finn2. Lane 1, molecular size markers (sizes, left margin). may be complex.

It will be useful to determine if other deletions exist in families in which $\Delta 9$ has been confirmed through RT-PCR or immunoblot experiments but in which splice-site mutations have not been found $^{3}$. It is also important to check for this deletion in families in which early onset disease occurs but in which missense mutations have not been found, and in families whose members present with spastic paraparesis. If this precise deletion or similar deletions occur in other non-related families, it may indicate that a specific underlying mutation mechanism exists.

Finally, in addition to the obvious change at the protein level, we cannot exclude the possibility that the deletion seen in $\Delta$ Finn also affects the transcriptional regulation, RNA stability or nonexon 9 splicing of PS1 in these families. Thus, there may be subtle phenotypic differences between $\Delta 9$ Finn and $\Delta 9$ splice site mutations.

Guy Prihar ${ }^{1}$, Auli VerkKoniemi ${ }^{2}$, Jordi Perez-Tur ${ }^{1,3}$, Richard Crook ${ }^{1}$, SARAH LinCOlN ${ }^{1}$, Henry Houlden ${ }^{1}$, Mirja Somer ${ }^{4}$, Anders Paetau ${ }^{5}$, HanNu Kalimo ${ }^{6}$, Andrew Grover ${ }^{1}$, LiIsA MYllykangas ${ }^{1,5}$, Mike Hutton ${ }^{1}$, JOHN HARDY $\&$ MatTi Haltia ${ }^{5}$

${ }^{1}$ Mayo Clinic, Jacksonville, 4500 San Pablo Road, Jacksonville, Florida 32224, USA ${ }^{2}$ Department of Clinical Neurosciences

${ }^{5}$ The Department of Pathology

Helsinki University Central Hospital FIN-00290, Finland

${ }^{3}$ Institut de Biomedicina de València, Unitat de Genètica Molecular, Jaume Roig, 11 València, E-46010 Spain

${ }^{4}$ The Family Federation of Finland,

P.O. Box 849

FIN-00101, Helsinki, Finland

${ }^{6}$ The Department of Pathology, University of Turku, Turku, FIN-20521, Finland

Email: hutton.michael@mayo.edu

1. Crook, R. et al. A variant of Alzheimer's disease with spastic paraparesis and unusual plaques due to deletion of exon 9 of presenilin 1. Nature Med. 4, 452-455 (1998).

2. Perez-Tur, J. et al. A mutation in Alzheimer's disease destroying a splice acceptor site in the presenilin 1 gene. NeuroReport 7, 204-207 (1995).

3. Kwok, J.B.J. et al. Two novel (M233T and R278T) presenilin 1 mutations in early onset Alzheimer's disease and preliminary evidence for association of presenilin 1 mutations with a novel phenotype. NeuroReport 8, 1537-1542 (1997). 